\title{
Skén\&graphie
}

SKIENaGAPHII Coulisses des arts du spectacle et des scènes

émergentes

$6 \mid 2019$

Voyages des cultures et des mémoires dans les arts

de la scène

\section{Dialogues avec les traditions de l'histoire : les régimes mémoriels des chorégraphes burkinabè}

Sarah Andrieu

\section{(2) OpenEdition}

12 Journals

Édition électronique

URL : https://journals.openedition.org/skenegraphie/2880

DOI : 10.4000/skenegraphie.2880

ISSN : 2553-1875

Éditeur

Presses universitaires de Franche-Comté

Édition imprimée

Date de publication : 1 novembre 2019

Pagination : 21-38

ISBN : 978-2-84867-673-9

ISSN : $1150-594 X$

Référence électronique

Sarah Andrieu, «Dialogues avec les traditions de l'histoire : les régimes mémoriels des chorégraphes burkinabè », Skén\&graphie [En ligne], 6 | 2019, mis en ligne le 01 juin 2021, consulté le 27 septembre 2021. URL : http://journals.openedition.org/skenegraphie/2880 ; DOI : https://doi.org/10.4000/ skenegraphie. 2880

Ce document a été généré automatiquement le 27 septembre 2021.

Presses universitaires de Franche-Comté 


\title{
Dialogues avec les traditions de l'histoire : les régimes mémoriels des chorégraphes burkinabè
}

\author{
Sarah Andrieu
}

1 On ne peut pas créer sans rétroviseurs ", « Pour savoir où l'on va, il faut savoir d'où l'on vient », «la tradition c'est notre base, sans elle nous n'existons pas». Ces phrases et leurs variantes ponctuèrent de nombreux entretiens et discussions au cours de mes recherches dans le monde de la création chorégraphique burkinabè'. L'idée que les danses traditionnelles ou plus généralement les savoirs et les imaginaires liés à la tradition constituent le point de départ à partir duquel le chorégraphe peut inventer un langage propre est, en effet, une pensée partagée par de nombreux danseurs et chorégraphes rencontrés à Ouagadougou. Néanmoins, si les débats et questionnements autour de la notion de tradition étaient centraux et assez consensuels dans les années 2000 alors que se structurait le monde transnational de la «danse contemporaine africaine $»^{2}$, ils prennent aujourd'hui des contours plus contrastés et polysémiques que les extraits d'entretiens qui ouvrent cette introduction.

2 En effet, si la place à accorder aux esthétiques, aux savoir-danser et aux imaginaires issus de la tradition est toujours au centre de certains débats, les questions liées à l'impérialisme des politiques culturelles françaises dans la structuration de ce mouvement chorégraphique et plus généralement celles touchant à l'hégémonisme du marché de l'art occidental, le souci porté aux dynamiques de professionnalisation ou encore les questions afférentes à la constitution d'un public et d'un marché local occupent désormais le devant de la scène. Plus exactement, ces enjeux actuels sont venus complexifier les processus créatifs et les discours ayant trait à la tradition en replaçant ceux-ci dans une réflexion plus globale sur la mémoire, l'identité et l'Histoire du continent dans son articulation au monde ${ }^{3}$.

3 Je souhaiterai dans cet article retracer certains de ces questionnements en décrivant les options singulières prises par certains créateurs burkinabè. Je tenterai ainsi de décrire les choix faits par certains danseurs de mobiliser, transformer, actualiser ou à l'inverse 
abandonner certains héritages gestuels en fonction de désirs et d'envies qui s'inscrivent au sein d'enjeux esthétiques et politiques contemporains. Il s'agira en définitive d'envisager les pratiques créatives mises en œuvre par certains chorégraphes burkinabè comme des lieux privilégiés pour envisager «les mécanismes qui font ressurgir le passé dans les polémiques sociales du temps présent $»^{4}$. Au fil de ce questionnement, un accent sera porté sur le tournant réflexif récent par lequel certains artistes s'attachent à articuler une pensée de la tradition, envisagée dans une optique essentiellement locale, à un questionnement portant sur l'Histoire de l'Afrique dans ses connexions au monde. Afin de saisir finement ces dynamiques, nous envisagerons deux pièces chorégraphiques intitulées " Tourments noirs » et « Zouhan » créées en 2009 et en 2012 par les chorégraphes Augustes Ouédraogo et Bienvenue Bazié ${ }^{5}$.

\section{Les régimes de traditionnalité des danseurs burkinabè}

$4 \mathrm{Si}$, pour la majorité des danseurs rencontrés à Ouagadougou, la tradition constitue un univers à forte charge émotionnelle et identitaire, admettre que l'ensemble des artistes burkinabè entretiendrait un rapport similaire à la tradition serait une erreur. Face à la diversité des mondes de la danse burkinabè et à la singularité des trajectoires individuelles et professionnelles, le chercheur se trouve davantage confronté à des "régimes de traditionnalité » hétérogènes plutôt qu'à un rapport consensuel à la tradition qui emprunterait des chemins chorégraphiques homogènes ${ }^{6}$. Néanmoins, si les « régimes de traditionnalité » sont différents d'un monde de la danse à un autre ${ }^{7}$ mais également pluriels à l'intérieur de chaque milieu chorégraphique, il s'avère possible de tracer des grandes lignes qui fédèrent les acteurs d'un même champ artistique. Ainsi, il demeure possible d'extraire certains axes structurant une manière commune de concevoir la tradition au sein de l'univers de la danse dite " contemporaine» ou «créative $»^{8}$, champ artistique qui retiendra plus particulièrement notre attention dans le cadre de cet article.

5 Nous pouvons constater, d'une part, que les artistes inscrits dans le champ de la «danse créative » ne conçoivent pas la performance uniquement comme un outil au service de la préservation ou de la valorisation des héritages chorégraphiques mais plutôt comme un mode d'expression individuel tourné vers une multiplicité d'enjeux (esthétiques, identitaires, politiques, sociaux...). Ainsi écrit le chorégraphe Salia Sanou, l'un des chefs de file de la création chorégraphique burkinabè :

Danser autrement. Telle pourrait être ma définition, simple, de la danse contemporaine. [...] Loin des questions de terminologie, l'essentiel n'est-il pas de comprendre comment notre mouvement est né et de quels récits il est porteur? Car c'est la vie d'aujourd'hui que nous dansons en choisissant ou non de la relier au passés.

6 En articulant le terme contemporain au temps présent et à "la vie d'aujourd'hui ", Salia Sanou met ici en débat la catégorie occidentale de "danse contemporaine », son usage hégémonique et les valeurs qui y sont liées ${ }^{10}$. L'autre facette importante de cette définition concerne le patrimoine et la possibilité, pour les créateurs africains, de s'en inspirer ou de s'en affranchir. D'une manière plus générale, il s'agit ici de refuser l'« ethnicisation » des pratiques créatives, posture partagée par de nombreux artistes non-occidentaux ${ }^{11}$, afin de se soustraire des identités assignées (celles de «danseurs contemporains africains» ou de "danseurs africains contemporains ») et d'affirmer une liberté artistique pleine et entière au même titre que les artistes européens et 
nord-américains. Cette posture est toutefois fragile, ambiguë et parfois impossible à tenir puisque l'origine géographique et culturelle de ces artistes est à la fois la source de leur stigmatisation et/ou de leur mise à l'écart des circuits de diffusions artistiques mondiaux $^{12}$ mais également le lieu de leur singularité esthétique et donc de leur inclusion au sein de ce même marché13.

C'est à l'intérieur de ces contraintes et enjeux paradoxaux que les artistes tissent leurs discours et leurs pratiques créatives autour des héritages gestuels et des imaginaires du passé. Lacina Coulibaly, artiste chorégraphique impliqué dans différents projets à Ouagadougou, et également enseignant dans diverses universités nord-américaines, explique à ce propos :

Moi, de toute façon, quand on parle de danse contemporaine, c'est ce que l'on crée aujourd'hui. Jusqu'à présent, j'ai du mal à voir ce qui fait que ça, c'est traditionnel, et ce qui fait que ça, c'est contemporain. Pour moi, même un pas traditionnel, je peux l'utiliser dans une création chorégraphique d'aujourd'hui. Si je trouve un sens pour moi, il n'y a pas de raison que ça ne marche pas. Tout ce que l'on crée aujourd'hui, je considère ça comme de la danse contemporaine. Même le pas traditionnel, je le prends, je le manipule d'une autre façon. Ou même je ne le manipule pas, je le danse comme il est, ça dépend de la situation que je crée dans ma chorégraphie, ça devient autre chose. [...] Pour moi contemporain, c'est aujourd'hui. Parce qu'on ne peut pas danser dans le futur. Pour moi, c'est dans le présent, ce qui est de nos jours. Parce que la danse traditionnelle évolue aussi : ce qui était dansé avant, nous on l'a changé et c'est sûr que la génération suivante, eux aussi ils vont le changer ${ }^{14}$.

Rejetant une vision romantique et nostalgique de la tradition qui fut au cœur des dynamiques de construction de l'imaginaire national depuis les années 1980, brisant l'idée que les "danses traditionnelles» doivent être mises en spectacle selon une prétendue « authenticité rurale » unique, les danseurs inscrits dans le mouvement de la « danse contemporaine » envisagent le vaste univers des traditions comme un domaine de ressources plurielles ayant toute sa place dans le présent. Les danseurs burkinabè partagent ainsi les deux modalités du discours sur la "modernité de la tradition" qu'évoque Hélène Neveu-Kringelbach à propos du monde chorégraphique sénégalais : la tradition comme "langue » et la tradition comme "critique sociale $»^{15}$. Le premier aspect, indique l'auteur, est contenu dans l'idée que ce qui fait l'intérêt et l'originalité des pratiques chorégraphiques africaines résident dans leur usage novateur de techniques gestuelles ancrées dans les patrimoines locaux. Le second aspect, poursuitelle, contient l'idée que la performance « traditionnelle » porte en elle un commentaire réflexif sur la vie sociale ${ }^{16}$. Cette double lecture de la tradition comme stock de gestes et d'imaginaires mais également réservoir d'idées sociales et politiques utiles aux artistes pour inscrire leur création dans l'actualité et les différentes «localités» qu'ils traversent au fil de leur carrières transnationales ${ }^{17}$ est bien partagée, nous le verrons, par les créateurs burkinabè.

\section{L'Histoire et les archives comme nouvelles ressources créatives}

9 Depuis une dizaine d'années, c'est également l'Histoire, celle des grands leadeurs politiques, des figures musicales $\mathrm{du}$ continent ou encore celle des empires précoloniaux, qui se trouve mobilisée par les créateurs burkinabè souhaitant s'extraire 
d'une vision romantique de "l'Afrique traditionnelle», partagée par certains publics africains et européens. Ce recours aux événements ou aux personnages historiques a également comme enjeu de reconnecter le continent africain à l'Histoire, un régime temporel que la pensée occidentale a longtemps réservé à l'Europe et l'Amérique du Nord $^{18}$. Pied de nez à un ancien président français estimant que l'homme africain n'était pas suffisamment entré dans l'histoire ${ }^{19}$, les créateurs d'Afrique sont de plus en plus nombreux à mobiliser les archives dans leurs créations rejoignant ainsi "l'impulsion archivistique ${ }^{20}$ " à l'œuvre dans les mondes globalisés de l'art contemporain. Comme plusieurs auteurs l'ont déjà montrée ${ }^{11}$, les artistes africains qu'ils soient musiciens, plasticiens, photographes ou danseurs - s'emparent aujourd'hui des archives pour proposer d'autres récits que les discours officiels construits par les États ou les narrations exotisantes élaborés en Occident. Ils élaborent ainsi de «nouvelles histoires » qui ont tout autant comme rôle de retracer autrement le passé que d'imaginer un présent et un futur alternatif ${ }^{22}$.

10 Assez peu présentes au tournant du millénaire lorsque le mouvement de la «danse contemporaine» se structure au Burkina Faso, les explorations des archives sont aujourd'hui au cœur de plusieurs projets chorégraphiques. Quittant l'univers atemporel de la tradition, les artistes s'attachent à remettre sur le devant de la scène les grands leadeurs et/ou militants d'Afrique dont les gestes, les discours ou les paroles collectés et re-composés sur scène doivent redonner, notamment à la jeunesse burkinabè à qui s'adressent plus particulièrement certains de ces spectacles, des raisons de croire en un avenir qui ne s'articule pas uniquement à des désirs de départ vers un ailleurs occidental. La diffusion de ces savoirs historiques vise également à déjouer certains imaginaires misérabilistes sur l'Afrique répandue chez certains spectateurs européens. C'est l'enjeu par exemple des pièces «Babemba» ou "Kalakuta Republik» du chorégraphe Serge Aimé Coulibaly qui se présentent comme des hommages aux grandes figures politiques, artistiques, sportives du continent ${ }^{23}$, mettant en scène l'image d'une Afrique forte et combative. La dernière pièce de ce chorégraphe, un opéra intitulé « Kirina ", du nom de la bataille ayant permis à Sundjata Keita de créer l'empire du Mali au XIII ${ }^{e}$ siècle, repose quant à elle sur des savoirs historiques, peu connus voir méconnus des publics européens mais également africains. Co-créée avec l'économiste et écrivain sénégalais Felwine Sarr et la chanteuse et compositrice malienne Rokia Traoré $^{24}$, cet opéra ne constitue pas aux yeux du chorégraphe une fresque chorégraphique inspirée du passé mais plutôt une «fantaisie du futur » mettant en scène l'histoire d'un peuple toujours en marche, prenant en main son destin. "Kirina ", indique Serge Aimé Coulibaly, ouvre ainsi une «fenêtre vers l'avenir » et permet de "regarder l'Afrique d'une autre manière ${ }^{25}$.

11 L'histoire, telle qu'elle se déploie dans certaines pièces chorégraphiques créées récemment par plusieurs chorégraphes burkinabè, vise en définitive à une « reprise en main » de récits du passé occultés ou détournés par le pouvoir colonial mais également par les états nations actuels. En les plaçant au cœur de leurs processus créatifs, en les connectant à d'autres mémoires faites de récits intimes ou familiaux contemporains, les artistes considèrent «l'extraction d'archives comme une manière de repenser, et, idéalement, de remodeler le présent et le futur ${ }^{26}$. Ainsi, ces dialogues avec le passé, nous le verrons plus précisément dans les lignes qui suivent, s'ils sont pluriels et prennent des formes hétérogènes, ont bien comme visée commune de faire bouger les regards, les discours et les imaginaires actuels. 


\section{« Tourments noirs » et « Zouhan » de la Compagnie Auguste-Bienvenue : dialogues avec l'histoire et les traditions}

12 Pour clore ce propos, je souhaiterai décrire les « jeux $»^{27}$ ou les dialogues avec l'histoire et les traditions réalisés par certains créateurs burkinabè. Afin de montrer comment ces dynamiques s'articulent entre elles plutôt qu'elles ne s'opposent, il me semble intéressant de mettre la focale sur deux pièces d'une même compagnie, la Compagnie Auguste-Bienvenue, qui, par ailleurs, propose des pièces où toutes références à l'histoire et à la tradition sont absentes. Avant d'entrer dans une brève ethnographie de ces pièces, revenons brièvement sur la trajectoire de leurs auteurs.

13 Nés au tournant des années 1980, Auguste Ouédraogo et Bienvenue Bazié ont fait ensemble leurs premiers pas aux Bourgeons, une des troupes de danses "néotraditionnelles" les plus réputées de Ouagadougou et ont connu ensemble leurs premières tournées nationales à l'adolescence. À partir de 1996, ils investissent les différentes formations en danse contemporaine proposées à Ouagadougou par l'Institut Français puis par les Rencontres Chorégraphiques Dialogues de Corps co-créées à la fin des années 1990 par les chorégraphes Salia Sanou et Seydou Boro ${ }^{28}$. Ils s'engagent alors dans un travail corporel distinct de celui incorporé au sein de la troupe des Bourgeons, espace créatif où les danses et musiques traditionnelles occupaient une large place ${ }^{29}$. À Ouagadougou, ils se forment notamment auprès de Christophe Cheleux, Nana Nilson, Jean-François Duroure, Robert Seyfried, Salia Sanou, Seydou Boro, Xavier Lot, Claude Brumachon et Benjamin Lamarche, Opiyo Okach, Gregory Makoma... Puis ils poursuivent leur apprentissage en France ${ }^{30}$ tout en s'engageant dans un travail de création au sein de la Compagnie Tâ. Certaines de leurs pièces, comme "Bûudu", seront remarquées par les professionnels et les premières tournées internationales débuteront dès 2003. Parallèlement à ces démarches créatives communes, chacun poursuivra un itinéraire plus solitaire. Bienvenue Bazié interprètera notamment le solo "Welcome to Bienvenue» créé par le chorégraphe français Xavier Lot tandis qu'Auguste collaborera avec Robert Seyfried sur la pièce " Transpace ».

14 Alors que les projets foisonnent et que les partenariats internationaux se multiplient, les deux chorégraphes ressentent le besoin de donner un sens renouvelé à leurs actions. Si le désir d'expérimentation artistique est toujours présent, la volonté de "passer à autre chose", de "faire les choses autrement" s'impose. La compagnie change alors de nom et devient la Compagnie Auguste-Bienvenue. Dans le sillage de leurs aînés, en particulier Salia Sanou et Seydou Boro, qui concomitamment à leur reconnaissance internationale ont initié des projets majeurs pour le développement de l'art chorégraphique au Burkina, Auguste et Bienvenue mettent en œuvre des actions dont le but est de faire jouer à la création chorégraphique son rôle dans le changement social. «Faire bouger les choses » à l'échelle locale devient un leitmotiv. En 2006, ils conçoivent "Traces", une pièce créée à partir des propositions de trois plasticiens français. Avec ce spectacle, les deux chorégraphes souhaitent sortir la danse contemporaine des murs des centres culturels français de Ouagadougou et BoboDioulasso afin de toucher un public différent. Ils tourneront dans différentes villes du pays, danseront sur des terrains de baskets, des stades de football, des centres culturels de quartiers. Ce désir d'ouvrir la danse contemporaine à de nouveaux acteurs se 
poursuivra avec la création en 2009 du projet « Engagement féminin », un programme de formation exclusivement destiné aux danseuses ouest-africaines visant à pallier le manque de femme dans ce champ professionnel en construction ${ }^{31}$. C'est à la lumière de cet engagement protéiforme qu'il nous semble intéressant d'appréhender les dialogues entrepris, par ces deux artistes, avec l'Histoire et les traditions.

\section{Des archives sonores pour nourrir le geste}

15 La première pièce qui retiendra notre attention est un solo, créé par Auguste Ouédraogo en $2009^{32}$ intitulé «Tourments noirs ». Prenant comme point de départ un questionnement large sur l'Afrique et la place que le continent doit occuper dans le contexte actuel de la globalisation, le solo s'appuie sur un discours prononcé par le président burkinabè Thomas Sankara à la tribune de l'ONU en 1984. Figure de la lutte anti-impérialiste, Thomas Sankara représente aujourd'hui un leader charismatique, pour une large partie de la jeunesse africaine. Alors que sur YouTube, les vidéos de ces discours cumulent aujourd'hui des millions de vues, l'image et la mémoire de ce héros de la révolution burkinabè assassiné en 1987, dans des circonstances toujours non élucidées ${ }^{33}$, furent longtemps gommées de l'imagerie officielle du régime de Blaise Compaoré. Et si la chute de Compaoré en 2014 a ouvert la voie à des commémorations publiques voire même à une véritable "Sankaramania " pour reprendre un terme utilisé par la presse locale, en 2009, lorsqu'Auguste Ouédraogo crée ce solo, la mémoire de Thomas Sankara est encore un sujet sensible dont l'usage public doit être réalisé avec précaution ${ }^{34}$.

16 Ainsi, la mise en scène et en gestes de ces archives orales permet de passer outre l'amnésie officielle et de redonner une existence publique à ce héros national qui, à l'époque, n'avait pas droit de cité dans les organes médiatiques officiels. À l'instar des musiciens de Libreville décrit par Alice Atérianus qui utilisent les technologies du sampling comme acte de résistance face à un État chronophage ${ }^{35}$, Auguste Ouédraogo s'attache à imaginer un nouveau récit de ce passé récent en mettant au centre de sa création, la pensée de cette figure politique tout autant occultée qu'adulée par une large partie des jeunes du continent.

Durant l'intégralité du solo, les mots de Thomas Sankara forment l'environnement sonore, tandis que le danseur fait résonner cet engagement dans son corps. On n'assiste dans cette pièce à aucune "reprise » de danses traditionnelles burkinabè, "l'identité de l'homme noir ", évoquée par l'ancien président burkinabè dans son discours, prend sur scène d'autres chemins corporels. Si le geste du poing gauche levé, symbole de la révolution sankariste, est repris à différents moments du solo, c'est davantage une énergie de l'engagement qui est mise en avant et donnée à voir à travers un corps en tension, des gestes saccadés, des chutes et des mouvements au sol. En mettant en scène une gestuelle de l'affirmation de soi, il insiste sur la nécessité de prendre ses responsabilités, d'accepter de "vivre africain ", «la seule façon de vivre libre et de vivre digne» selon les mots de Sankara ${ }^{36}$. Ainsi, si le propos et l'environnement sonore de "Tourments Noirs" font référence à un imaginaire identitaire ancré dans le national voire le continental, le langage chorégraphique proposé dans ce solo relève plus d'un bagage chorégraphique composite et cosmopolite, résultat d'une trajectoire de formation hétérogène que d'un habitus corporel localisée ${ }^{37}$. Tout en s'inscrivant clairement dans une gestuelle personnelle et détachée de toutes 
attaches « ethniques » ou "nationales », le chorégraphe incarne un récit collectif qui regroupe une partie de plus en plus large de la jeunesse ouest-africaine autour des valeurs d'engagement, d'autonomie et de rupture.

\section{Ré-enchanter la tradition : toucher de nouveaux publics}

La seconde pièce que nous souhaitons évoquer s'intitule "Zouhan", mot lyélés8 qui signifie la parole. Cette performance transdisciplinaire, créée par Auguste Ouédraogo et Bienvenue Bazié en 2012 est avant tout conçue comme un spectacle participatif au sein duquel le public est amené à devenir la population d'un village imaginaire. Pensé pour l'espace public, ce spectacle repose sur l'idée d'une "transposition» des veillées de contes où petits et grands se retrouvent la nuit pour écouter les histoires des anciens. Sans folklorisme ni passéisme, la pièce est tout entière inspirée de langages expressifs traditionnels - le conte, la musique, le chant et la lutte - dont les résonances émotionnelles et corporelles sont encore particulièrement vives chez le public burkinabè. Accompagné par le joueur de Tama et de Kora Arouna Coulibaly, le conteur Kientega Pingdewindé Gérard, plus connu à Ouagadougou sous le surnom de KPG, incarne dans la pièce le rôle du griot, le généalogiste et historien coutumier. Il a comme rôle d'embarquer le public dans cet univers onirique en le guidant vers les différents sites de la déambulation. Après plusieurs arrêts devant différents symboles du village imaginaire, dont "l'histoire millénaire " est narrée par KPG, chacun est invité à s'asseoir à terre en demi-cercle autour d'un espace délimité par de petites lampes à pétroles. Le griot et le musicien rejoignent la chanteuse et danseuse Mariam Pale à l'une des extrémités du cercle. Au centre, Auguste et Bienvenue, les deux lutteurs du conte se préparent au combat. Les deux protagonistes du combat, "Silam Silam » du village de Kamsonghin et "Tao Tao» du village d'Arbolés9, font l'objet d'une présentation épique par le griot avant que la danse ne commence.

La chorégraphie proposée par Auguste et Bienvenue est toute entière inspirée des gestes des lutteurs, une pratique vive et populaire au Burkina Faso. Néanmoins, il ne s'agit pas d'une transposition stricto sensu d'une scène de lutte traditionnelle dans un cadre spectaculaire. Si les deux danseurs s'approprient la posture des lutteurs (jambes demi-pliés, dos droit, bras et regard tendus vers l'adversaire), les gestes sont transformés, dé-composés, ralentis et les corps à corps se transforment régulièrement en portés plutôt qu'en attaques frontales visant à mettre l'adversaire au sol. Cette « lutte dansée », selon la formule des chorégraphes, s'inspire du langage corporel de la lutte mais le trans-forme dans une chorégraphie où sont convoqués des mouvements issus de différents répertoires, notamment ceux dans lesquels ont été formés Auguste et Bienvenue: certaines danses traditionnelles burkinabè et certaines techniques de danse contemporaine occidentale.

20 Les différentes techniques et les imaginaires issus de la tradition et convoqués dans Zouhan visent ainsi tout autant à explorer des espaces scénographiques et émotionnels nouveaux qu'à renouveler le rapport au public et espérer ainsi toucher de nouveaux spectateurs. Dans un contexte où la « danse contemporaine » est souvent méconnue et ou mal perçue des populations burkinabè qui estiment que cette danse s'éloigne trop des réalités esthétiques et sociales locales, la tradition ré-enchantée dans Zouhan sert à re-connecter entre eux différents univers (celui de la veillée et du spectacle scénique 
notamment) afin de d'impliquer un public burkinabè. Les imaginaires et esthétiques servent au final à faire advenir un «temps de partage $»^{40}$ au sein duquel artistes et spectateurs se trouvent réunis le temps du spectacle.

21 Après avoir imaginé "Zouhan ", Auguste Ouédraogo et Bienvenue Bazié créent la pièce «PerformerS » en 2015 au sein de laquelle ils collaborent avec un ou une musicien(ne) présent(e) sur scène. Dans cette pièce, aucune référence à l'histoire ou la tradition, mais une volonté de travailler les techniques d'improvisations et les manières dont les corps peuvent (ré)agir dans l'instant devant un public. Ici, les langages chorégraphiques ne s'inspirent guère de gestes issus de la tradition mais plutôt d'un bagage corporel composite et singulier forgé au fil du parcours individuel de chacun des artistes. Enfin, en 2017, Bienvenue Bazié se lance dans la création d'un solo intitulé «Peubléto-rêves et réalités » au sein duquel il explore non pas l'Histoire mais sa propre histoire, notamment son choix de faire de la danse son métier dans un environnement social peu propice. Il collabore avec le vidéaste Grégory Hiétin qui mène des entretiens filmés à Ouagadougou avec les parents de Bienvenue, images diffusées sur scène durant la performance. Ici le chorégraphe produit et s'appuie sur des archives mais celles-ci demeurent avant tout intimes et familiales.

Les propositions chorégraphiques de la Compagnie Auguste-Bienvenue permettent d'entrevoir de nouvelles postures artistiques au sein desquelles s'articulent volonté de proposer d'autres récits du passé mais également du présent, de toucher des publics internationaux mais également locaux et au final d'être libéré de certaines contraintes esthétiques et identitaires pour créer des œuvres singulières dont la tradition et l'Histoire représentent certes des sources d'inspirations importantes mais non des carcans dont les artistes seraient prisonniers. En outre, lorsque Tradition et Histoire se trouvent au cœur du processus créatif, les techniques corporelles et les imaginaires issus du passé se trouvent re-composés à l'aune des trajectoires professionnelles (notamment au sein du monde glofredbalisé de la danse contemporaine) et personnels des artistes. Enfin, ces dialogues avec le passé renouvellent également les enjeux nationalistes ou régionalistes qui, encore aujourd'hui, guident le travail de nombreuses troupes de danses néo-traditionnelles burkinabè tout en continuant d'inscrire l'acte chorégraphique dans un contexte politique contemporain. Les artistes chorégraphiques burkinabè évoqués dans cet article ne souhaitent guère en effet déconnecter la danse des enjeux sociaux et politiques actuels. En articulant la création chorégraphique à des événements ou à des récits historiques, ils travaillent au contraire à ancrer leur pratique chorégraphique dans le monde contemporain. Ce faisant, ils s'inscrivent tout autant au sein des "nouvelles tendances documentaires" qui animent le monde globalisé de la création chorégraphique ${ }^{41}$ que dans la généalogie plus longue de "l'art engagé » qui structure une partie des dynamiques artistiques burkinabè depuis les années 1980. 


\section{NOTES}

1. Ces recherches ont été menées entre 2003 et 2007 dans le cadre de ma thèse de doctorat puis, à partir de 2010, au sein des programmes ANR « Système de savoirs et d'apprentissage en Afrique » coordonné par Bruno Martinelli et Pascale Maïzi et «Création musicale, circulation et marché d'identités en contexte global » coordonné par Emmanuelle Olivier. Ces enquêtes de terrain se sont principalement déroulées à Ouagadougou au sein de différentes institutions artistiques nationales (Institut national de formation artistique et culturelle; Ballet National ; Ministère de la culture), de festivals (Semaine National de la Culture, Rencontres chorégraphiques de Ouagadougou Dialogues de Corps) et d'espaces de formation (Centre de développement chorégraphique La Termitière, formation professionnelle «Engagement Féminin»). Ces recherches longitudinales et multi-sites (entre le Burkina Faso et la France notamment) ont permis de retracer des trajectoires professionnelles complexes et d'engager des discussions riches et suivies avec certaines danseuses et certains danseurs.

2. Sur l'histoire du mouvement de la «danse contemporaine africaine» du point de vue de la politique de coopération culturelle française, se rapporter notamment aux travaux d'Altaïr Despres et d'Annie Bourdié (Altaïr Despres, Se faire contemporain. Les danseurs africains à l'épreuve de la mondialisation culturelle, Publications de la Sorbonne, Paris, 2016 ; Annie Bourdie, "Art chorégraphique contemporain d'Afrique, enjeux d'une reconnaissance », Marges 16, 2013, p. 7386). Pour une lecture localisée de la fabrique de cet art global voir Hélène Neveu Kringelbach pour le contexte sénégalais (Hélène Neveu Kringelbach, Dance Circles. Movement, Morality and SelfFashioning in Urban Senegal, New York, Oxford, Berghahn Books, 2013) ainsi que les travaux de Nadine Sieveking (Nadine Sieveking, «La localité de l'art global. Danser au contemporain à Ouagadougou », in Sarah Andrieu \& Emmanuelle Olivier (dir.), Création artistique et imaginaires de la globalisation, Hermann, Paris, 2017, p. 255-282) et mes propres recherches sur le Burkina Faso (Sarah Andrieu, «Les valeurs de la création chorégraphique ouest-africaine ", Volume! La revue des musiques populaires, vol.10-1, 2014, p. 89-112). Sur une généalogie sensible qui accorde une large place aux souvenirs personnels ainsi qu'aux vécus et aux points de vue des danseurs, je renvoie à l'ouvrage de Salia Sanou (Salia Sanou, Afrique. Danse contemporaine, Paris, Cercle d'art/ Centre National de la Danse, 2008).

3. Signalons que ces réflexions ne sont pas spécifiques au monde cosmopolite de la «danse contemporaine africaine " mais partagées par de nombreux créateurs et intellectuels africains militant pour penser l'Afrique dans ses relations au monde. Voir notamment Achille MBEMBE \& Felwine SARR (dir.), Écrire l'Afrique-Monde, Paris, Édition Philippe Rey, 2017.

4. Jean COPANS, La Longue marche de la modernité africaine : savoirs, intellectuels, démocratie, Paris, Éd. Karthala, 1990, p. 130.

5. Je tiens à remercier chaleureusement ces deux artistes ainsi que les danseuses et danseurs cités dans cet article pour l'accueil bienveillant fait à mes recherches. Je remercie également Nadine Sieveking avec qui j'ai le plaisir de mener des enquêtes de terrain, d'échanger et d'écrire sur la création chorégraphique burkinabè depuis 2010.

6. L'expression et l'idée qui sous-tendent la notion de « régime de traditionnalité » sont inspirées des travaux de François Hartog sur les «régimes d'historicité " (François HARToG, Régimes d'historicité. Présentisme et expérience du temps, Paris, Le Seuil, 2003).

7. Pour une description détaillée des différents "mondes de la danse » scéniques burkinabè et des régimes de traditionnalité qui s'y rapportent, je me permets de renvoyer à mes travaux de recherche doctorale. Sarah ANDRIEU, Le Spectacle des traditions. Analyse anthropologique du processus de spectacularisation des danses au Burkina Faso, thèse de doctorat en anthropologie, Université AixMarseille 1, soutenue le 10 décembre 2009. 
8. Les expressions «danse contemporaine africaine » ou «danse africaine contemporaine » ont fait l'objet d'intenses débats et de rejets par certains chorégraphes africains ne souhaitant pas que leur danse soit considérée comme une branche exotique de la «danse contemporaine occidentale ». Au Burkina Faso, Salia Sanou et Seydou Boro préfèrent parler de " danse créative » ou de «danse d'aujourd'hui ». Ainsi, l'expression "danse contemporaine », si elle est souvent conservée par les danseurs burkinabè, n'est pas envisagée au sein d'une filiation unique à l'art contemporain occidental, mais plutôt en référence à l'actualité et à la co-temporalité que partagent les chorégraphes africains avec leurs homologues du Nord. Pour une analyse fine de ces dynamiques, se rapporter à Nadine SIEVEKING, op. cit.

9. Salia SANOU, op. cit., p. 28.

10. Pour une analyse fine des processus de «(dé)catégorisations » et de "re-catégorisations » effectués par les danseurs ouest-africains, se rapporter aux travaux de Mahalia Lassibille, notamment Mahalia LASSIBILLE, "Quand la catégorie (dé)catégorise. Méthodologie autour du processus d'interprétation du «contemporain » en danse au Niger », in Federica FRATAGNOLI \& Mahalia LASSIBILLE, Danser contemporain. Gestes croisés d'Afrique et d'Asie du Sud, Deuxième époque, Montpellier, 2018, p. 287-316.

11. Nadine SIEVEKING, op. cit; Hans BELTING, « Contemporary Art as Global Art: A Critical Estimate », in Hans Belting \& Andrea BUdDEnSiEg (eds.), The Global Art World. Audiences, Markets, and Museums, Ostfildern, Hatje Cantz, 2009, p. 38-73.

12. Kasfir Sidney LITTLEFIELD, L'Art contemporain africain, Paris, Éditions Thames and Hudson, 2000.

13. Altaïr DESPRES, «L'Afrique appropriée. Visibilité et légitimation de l'Afrique dans le champ de la danse contemporaine ", Hommes et migrations, n 1297, mai-juin 2012, p. 116-126.

14. Entretien réalisé avec Lacina Coulibaly à Ouagadougou le 30 juillet 2011.

15. Hélène NEVEU KRINGELBACH, op. cit, p. 179.

16. Id.

17. Signalons que pour les danseurs rencontrés, le « local » fait surtout référence au Burkina Faso mais il peut également être multi-situé et suivre les espaces que ces derniers habitent ou traversent. Ainsi, le «local» peut faire référence à Ouagadougou mais également à Paris, Montpellier, Bordeaux, Berlin, Bruxelles ou New York pour citer certaines villes où résident, une partie du temps, les chorégraphes évoqués dans cet article.

18. Comme l'a bien montré Johannes Fabian, l'anthropologie a émergé en tant que «discours allochronique " situant l'Autre "observé» dans une temporalité distincte de celle des " observateurs ». Les études africaines de la fin du XIX siècle ont ainsi enfermé le continent dans une vision exotique où l'histoire n'avait pas de place. Aujourd'hui encore, l'idée que l'Afrique constitue un continent "sans" histoire (mais également "sans écritures", "sans système politique organisé ») mais "avec» des coutumes et des traditions se donne à entendre en Occident. Johannes Fabian, Le Temps et les autres. Comment l'anthropologie construit son objet, Toulouse, Anacharsis, 2006.

19. Il s'agit du tristement célèbre "Discours de Dakar» prononcé par Nicolas Sarkozy, le 26 juillet 2007 à l'Université Cheik Anta Diop au sein duquel l'ancien président français proclamait: «Le drame de l'Afrique c'est que l'homme africain n'est pas assez entré dans l'histoire... ». Ce discours a provoqué plusieurs écrits stimulants notamment l'ouvrage collectif dirigé par l'historienne Adame Ba Konaré (Adame BA KONARÉ (dir.), Petit Précis de remise à niveau sur l'histoire africaine à l'usage du président Sarkozy, Paris, La découverte, 2008).

20. Hal FOSTER, « An Archival Impulse », October, vol. 110, 2004, p. 3-22.

21. Voir notamment ces deux publications collectives récentes: Maëline Le Lay, Dominique MALAQUAIS \& Nadine SIEGERT (dir.), Archive (re)mix : vues d'Afrique. Rennes, Presses universitaires de Rennes, 2015 ; Alice ATERIANUS-OWANGA et Nora GRÉANI, « ArTchives ", Gradhiva, n² 24, 2016.

22. Maëline LE LAY, Dominique MALAQUAIS et Nadine SIEGERT, op. cit, p. 15. 
23. Dans «Babemba ", Serge Aimé Coulibaly met en scène différentes figures des luttes anticoloniales ou anti-apartheid (Patrice Lumumba, Thomas Sankara, Kwamé Nkrumah, Nelson Mandela, mais aussi Samory Touré et Shaka Babemba) tandis que « Kalakuta Republik » est une pièce construite autour de l'œuvre musicale, de l'univers nocturne et de l'engagement de Fela Anikulapo Kuta, musicien et activiste nigérian.

24. La première représentation de cet opéra a eu lieu au Festival de Marseille le 29 juin 2018.

25. Les citations sont extraites de la présentation vidéo de la pièce faite par Serge Aimé Coulibaly et disponible sur le site du Festival de Marseille. http://www.festivaldemarseille.com/fr/fr/lesartistes/serge-aime-coulibaly

26. Maëline LE LAY, Dominique MALAQUAIS \& Nadine SIEGERT, op. cit., p. 16

27. L'emploi de ce terme fait référence à l'article d'Yvonne Hardt qui, à plusieurs égards, éclaire certaines des dynamiques que j'ai pu observer au Burkina Faso dans le champ de la création chorégraphique. Si, la "danse contemporaine occidentale » est bien "locale " et "localisée », comme l'explique l'auteur de cet article, elle forme également un réseau trans-local au sein duquel circulent certaines normes et valeurs. Yvonne HARDT, « Staging the Ethnographic of Dance History : Contemporary Dance and Its Play with Tradition », Dance Research Journal, $n^{\circ} 43(1), 2011$, p. 27-42.

28. Nés à la fin des années 1960, Salia Sanou et Seydou Boro ont évolué durant de nombreuses années, dans des compagnies de danses traditionnelles et de théâtre à Bobo-Dioulasso et à Ouagadougou avant d'entamer une carrière internationale au début des années 1990 notamment auprès de Mathilde Monnier au centre chorégraphique de Montpellier. En parallèle à cette carrière, ils s'investiront fortement dans la structuration du champ chorégraphique au Burkina Faso en créant le festival «Dialogues de corps » puis le Centre de Développement chorégraphique de Ouagadougou La Termitière en 2006. Pour plus de détails sur ces deux figures de la création chorégraphique, voir le livre de Salia Sanou (2008, op. cit.) ainsi que l'ouvrage de Gérard Mayen qui leur est consacré (Gérard Mayen, Danseurs contemporains du Burkina Faso. La compagnie Salia nï Seydou au temps de la mondialisation, Paris, L'Harmattan, 2006).

29. Notons que les savoir-danser traditionnels qui sont au cœur du répertoire de cette troupe constitue une "tradition chorégraphique parallèle " (Anthony SHAY, Choreographic Politics. Sate Folk Dance Companies: Representation and Power, Wesleyan University Press, Middletown [ÉtatsUnis], 2002) qui a déjà connu de multiples transformations formelles depuis son "extraction » des contextes rituelles ou festifs locaux. Ces répertoires de danse «néo-traditionnelles » se sont essentiellement constitués au début des années 1980 durant la politique culturelle dirigiste de la Révolution démocratique et populaire burkinabè.

30. Ces formations prennent place notamment dans le cadre des « Ateliers du monde » proposés dans le cadre du festival Montpellier danse mais également au Centre chorégraphique de Nantes alors dirigé par Claude Brumachon et Benjamin Lamarche.

31. Pour plus de détails sur le programme Engagement Féminin, voir Sarah ANDRIEU \& Nadine SIEVEKING, «"Faire bouger les choses!" Engagement féminin et dynamiques sociales de la danse contemporaine en Afrique", Africultures. La revue des cultures africaines, 2012. http:// africultures.com/faire-bouger-les-choses-11290/?

utm_source=newsletter\&utm_medium $=$ email\&utm_campaign $=429$

32. Bienvenue Bazié travaillera également sur cette création en qualité d'assistant à la chorégraphie.

33. Voir à ce sujet l'article de René ОТАҮЕК, « Assasinat de Thomas Sankara : enfin la vérité ?» paru le 13 décembre 2017 dans Le Monde. https://www.lemonde. fr/afrique/article/2017/12/13/ assassinat-de-thomas-sankara-enfin-la-verite_5229137_3212.html

34. Le chanteur burkinabè Smockey, qui avait proposé en novembre 2009 un morceau et un clip vidéo intitulé «À qui profites le crime? » articulant chanson originale, samples de discours d'anciens responsables politiques (François Mitterrand, Blaise Compaoré, Thomas Sankara...) et 
images d'archives, reçut de multiples menaces. Le clip fut également censuré de la télévision publique. https://www.youtube.com/watch ?v =9Vloo5GD2K8

35. Alice ATÉRIANUS, "Sampler les bruits de la ville, archiver les traces des balles. La création musicale hip-hop contre les politiques de l'amnésie au Gabon ", Gradhiva, n 24, p. 108-135.

36. Les passages entre guillemets sont extraits de la note d'intention du spectacle.

37. À ce titre, il est intéressant de noter que le seul geste qui s'inscrit explicitement dans un habitus corporel que l'on pourrait qualifier de national, le poing gauche levé, est en fait transnational puisqu'il est le symbole gestuel de nombreux activistes d'extrêmes gauches de par la monde.

38. Les Lyélé constituent une population du centre-ouest du Burkina Faso dont est originaire Bienvenue Bazié.

39. Si le village re-créé dans Zouhan ainsi que les personnages qui l'habitent sont imaginaires, Kamsonghin et Arbolé tirent leur nom de deux lieux réels. Le premier est le nom d'un quartier de la capitale et le second, le village natal de KPG, est situé à $70 \mathrm{~km}$ de Ouagadougou.

40. Dans le dossier de présentation du spectacle, «Zouhan» est présenté comme "une proposition artistique nourrie de la mémoire, de l'ouverture, des valeurs culturels et éducatives. Un temps de rencontre entre la musique, la danse, le conte C'est aussi une proposition d'échanger directement avec le spectateur. Un temps de partage et de transmission » (voir http://cieauguste-bienvenue.blog4ever.com).

41. Frédéric Pouillaude, «Danse as documentary: Conflictuel Images in the Choreographic Mirror (On Archive by Arkadi Zaides) », Dance Research Journal, vol. 48, Issue 2, 2016, p. 80-94.

\section{AUTEUR}

\section{SARAH ANDRIEU}

Anthropologue, maîtresse de conférences au département des Arts, section danse, de l'université Nice-Sophia Antipolis. Elle est membre du Centre Transdisciplinaire d'Epistémologie de la Littérature et des Arts vivants (CTEL) et membre associée à l'Institut des mondes africains (IMAF). Ses recherches, menées au Burkina Faso depuis 2002, portent d'une part sur les usages politiques des danses traditionnelles par l'état postcolonial et d'autre part sur les dynamiques de circulation d'esthétiques, de savoirs, d'imaginaires dans le champ globalisé de la « danse africaine contemporaine ». Elle a récemment coordonné avec Emmanuelle Olivier, l'ouvrage collectif Création artistique et imaginaires de la globalisation (Edition Hermann, 2017). Elle est membre du comité scientifique éditorial de la revue Recherches en danse. 